\title{
Serial Changes in Cardiac Sympathetic Nervous Function After Transcatheter Aortic Valve Replacement: A Prospective Observational Study Using 123I-meta-iodobenzylguanidine Imaging
}

\section{Yoshito Kadoya ( $\nabla$ m03020kdy@gmail.com )}

Graduate School of Medical Science, Kyoto Prefectural University of Medicine https://orcid.org/00000001-8333-2219

\section{Kan Zen}

Kyoto Prefectural University of Medicine: Kyoto Furitsu Ika Daigaku Nagara Tamaki

Kyoto Prefectural University of Medicine: Kyoto Furitsu Ika Daigaku

\section{Shunsuke Nakamura}

Kyoto Prefectural University of Medicine: Kyoto Furitsu Ika Daigaku

\section{Tomoki Fujimoto}

Kyoto Prefectural University of Medicine: Kyoto Furitsu Ika Daigaku

\section{Masaki Yashige}

Kyoto Prefectural University of Medicine: Kyoto Furitsu Ika Daigaku

\section{Kazuaki Takamatsu}

Kyoto Prefectural University of Medicine: Kyoto Furitsu Ika Daigaku Nobuyasu Ito

Kyoto Prefectural University of Medicine: Kyoto Furitsu Ika Daigaku

\section{Michiyo Yamano}

Kyoto Prefectural University of Medicine: Kyoto Furitsu Ika Daigaku

Tetsuhiro Yamano

Kyoto Prefectural University of Medicine: Kyoto Furitsu Ika Daigaku

Takeshi Nakamura

Kyoto Prefectural University of Medicine: Kyoto Furitsu Ika Daigaku Hidetake Kawajiri

Kyoto Prefectural University of Medicine: Kyoto Furitsu Ika Daigaku

\section{Satoshi Numata}

Kyoto Prefectural University of Medicine: Kyoto Furitsu Ika Daigaku

\section{Hitoshi Yaku}

Kyoto Prefectural University of Medicine: Kyoto Furitsu Ika Daigaku

\section{Satoaki Matoba}




\section{Research Article}

Keywords: 123I-meta-iodobenzylguanidine imaging, cardiac sympathetic nervous function, aortic stenosis, transcatheter aortic valve replacement

Posted Date: April 26th, 2021

DOl: https://doi.org/10.21203/rs.3.rs-452927/v1

License: (c) (1) This work is licensed under a Creative Commons Attribution 4.0 International License. Read Full License 


\section{Abstract}

\section{Purpose:}

Transcatheter aortic valve replacement (TAVR) can rapidly improve cardiac sympathetic nervous function (CSNF) within 2 weeks in patients with aortic stenosis (AS). However, it remains unclear whether such short-term improvements will be sustained thereafter. The present study aimed to investigate the midterm (i.e., 6-12 months) effects of TAVR on CSNF in patients with severe AS using ${ }^{123}$ I-metaiodobenzylguanidine (MIBG) imaging.

\section{Methods:}

Patients with severe AS who were scheduled to undergo TAVR between October 2017 and June 2019 were enrolled in this single-centre, prospective, observational study. MIBG imaging was performed at baseline, within 2 weeks after TAVR, and at 6-12 months post-TAVR to evaluate the heart-mediastinum ratio $(H / M)$ and washout rate (WR). Differences between each MIBG parameter at three time points were analysed, and factors involved in the long-term improvement in the late $\mathrm{H} / \mathrm{M}$ were investigated.

\section{Results:}

Of 183 consecutive patients, 75 (19 men; median age: 86 years) were evaluated. The late $\mathrm{H} / \mathrm{M}$ significantly improved within 2 weeks after TAVR $(P=0.041)$ and further improved over $6-12$ months after $\operatorname{TAVR}(P=0.041)$. The WR rapidly improved immediately after TAVR $(P=0.003)$ but remained unchanged at 6-12 months $(P=0.827)$. Multivariate analysis revealed that the baseline mean aortic valve pressure gradient ( $\mathrm{mPG}$ ) was an independent predictor of mid-term improvement in the late $\mathrm{H} / \mathrm{M}(>0.1)$ (adjusted odds ratio: $0.035 ; 95 \%$ confidence interval: $0.004-0.070 ; P=0.037$ ). Patients with a high baseline $\mathrm{mPG}$ $(\geq 58 \mathrm{mmHg}$ ) exhibited a significantly greater increase in the late $\mathrm{H} / \mathrm{M}$ than those with a low baseline $\mathrm{mPG}(<42 \mathrm{mmHg})(0.24$ vs. $0.01 ; P=0.029)$.

\section{Conclusions:}

CSNF, as denoted by the late $\mathrm{H} / \mathrm{M}$, demonstrated sustained improvement from within 2 weeks after TAVR until 6-12 months later. Such improvement was related to baseline hemodynamic AS severity.

\section{Introduction}

Aortic stenosis (AS) is one of the leading causes of cardiac morbidity and mortality among elderly patients [1]. While surgical aortic valve replacement is considered as the established treatment for severe symptomatic AS, transcatheter aortic valve replacement (TAVR) is another important treatment option, particularly for elderly adults or patients with prohibitive surgical risk [2].

Patients with AS exhibit impairments in cardiac sympathetic nervous function (CSNF), and several studies have indicated improvements in CSNF consequent to TAVR [3, 4]. Our previous research 
demonstrates that CSNF, as assessed by ${ }^{123}$-meta-iodobenzylguanidine (MIBG) imaging, significantly improved within a short duration, approximately 1 week, after TAVR [4]. However, whether such short-term improvement in CSNF will be sustained thereafter remains unclear.

Functional improvement in cardiac sympathetic nerve endings is generally thought to require substantial time (i.e., more than a few months). Previous studies on the effects of beta-blockers or angiotensinconverting enzyme inhibitors (ACEIs) evaluated changes in MIBG parameters, including the heartmediastinum ratio $(\mathrm{H} / \mathrm{M})$ and washout rate $(\mathrm{WR})$, over a period of $6-12$ months $[5,6]$. To evaluate the effects of TAVR on CSNF adequately, it is necessary to observe not only the short-term changes in the indices of CSNF, but also long-term changes; whether or not TAVR-dependent early improvement is transitory or sustainable should be examined. Therefore, the present study aimed to investigate the midterm effects of TAVR on CSNF using serial cardiac MIBG imaging.

\section{Material And Methods}

\section{Study Population}

This single-centre, prospective, observational study enrolled consecutive patients who were scheduled to undergo TAVR for severe AS between October 2017 and June 2019. Severe AS was defined according to the current American College of Cardiology/American Heart Association guidelines [2]. Cardiac MIBG imaging was planned to be performed at baseline, within 2 weeks after TAVR, and at 6-12 months after TAVR to evaluate the H/M and WR. All baseline and procedural data, including laboratory and echocardiographic findings, were prospectively collected at the same time as MIBG scintigraphy. Echocardiographic measurements during the study period were conducted in accordance with the recommendations of the American Society of Echocardiography.

The exclusion criteria for the initial MIBG evaluation of patients were as follows: (a) active cancer and/or Parkinson's disease; (b) incapability to perform MIBG imaging due to severe dementia and/or mental disorder; (c) ongoing therapy with medications known to interact with MIBG (e.g. labetalol, reserpine, tricyclic antidepressants, sympathomimetic amines, serotonin-norepinephrine reuptake inhibitors) [7]; (d) utilization of trans-apical and trans-aortic TAVR or of valve-in-valve TAVR for structural valve deterioration in bioprosthetic aortic valves; (e) unstable preprocedural conditions (e.g. cardiogenic shock) treated with intravenous catecholamine administration; ( $f$ ) insufficient MIBG imaging quality; and (g) refusal to provide consent for study participation. Patients with serious procedure-related complications (e.g., left ventricular perforation, annular rupture, coronary obstruction, cardiac tamponade) were excluded from the subsequent postoperative MIBG evaluation. Furthermore, patients treated with newly added beta-blockers, ACEls, and/or angiotensin II receptor blockers after TAVR were excluded from the final MIBG evaluation.

All procedures were performed in accordance with the ethical standards of the institutional research committee of Kyoto Prefectural University of Medicine (ERB-C-1081-3) and with the 1964 Declaration of Helsinki and its later amendments or comparable ethical standards. Informed consent was obtained from 
all participants. The corresponding author (K.Z.) had full access to all data in the study and takes responsibility for data integrity as well as data analyses.

\section{Cardiac MIBG Imaging}

The first MIBG imaging was performed at the time of admission for TAVR, which was a few days prior to TAVR in most cases. The second MIBG imaging was carried out at $>2$ days post-TAVR and when patients' conditions were stable in order to avoid the influence of perioperative transfusion or infection. In our institution, all patients were routinely followed up at 1, 6, and 12 months after TAVR. The third MIBG imaging was performed at the 6-month follow-up; depending on patients' availability, the third MIBG imaging was also permitted to be conducted at the 12-month follow-up.

MyoMIBG (FUJIFILM RI Pharma Co. Ltd., Tokyo, Japan) was intravenously administered at a dose of 111 MBq. Anterior planar images were acquired at 15 min (early image) and $240 \mathrm{~min}$ (late image) after MIBG injection using a large-field-of-view gamma camera (Discovery NM/CT 670; GE Healthcare, Waukesha, WI, USA) equipped with a medium-energy collimator. To obtain semi-quantitative parameters for tracer distribution, MIBG imaging analysis was performed based on the region of interest using smart MIBG software (FUJIFILM RI Pharma Co. Ltd., Tokyo, Japan), which was developed to semi-automatically determine the $\mathrm{H} / \mathrm{M}$ and correct the $\mathrm{H} / \mathrm{M}$ to standard medium-energy collimator conditions [8]. The $\mathrm{H} / \mathrm{M}$ was determined by measuring the average counts in each region, whereas the WR from the heart was calculated as the time decay-corrected difference between early and late images. Experienced radiology technicians who were unaware of patients' information conducted all analyses.

\section{TAVR procedure}

The TAVR procedure was performed using the transfemoral or trans-subclavian approach under general anaesthesia or conscious sedation with local anaesthesia. Either the Edwards SAPIEN 3 (Edwards Lifesciences, Irvine, CA, USA) or Medtronic CoreValve Evolut R/Pro (Medtronic, Minneapolis, MN, USA) was used as a prosthesis. The multidisciplinary heart team determined the indication, valve type, and/or vascular access for TAVR.

\section{Statistical Analysis}

Normality of distributions was assessed using the Shapiro-Wilk test. Categorical variables are presented as counts and percentages, whereas continuous variables are expressed as median (25th-75th percentile) given that few variables followed a normal distribution. With respect to normally distributed variables, the median and mean values were nearly identical. Depending on variable distribution, Friedman's test or repeated-measures analysis of variance (ANOVA) was performed for the comparison of laboratory, echocardiographic, and MIBG findings at the three time points. In case of significance, pairwise post hoc tests with Holm correction were performed. Multiple regression analysis was conducted to determine the predictors of improvement in the late $\mathrm{H} / \mathrm{M}$ at 6-12 months after TAVR versus baseline. Following our previously established methodology, the cut-off values for the improvement in the $\mathrm{H} / \mathrm{M}$ and WR were set at 0.1 and $3 \%$, respectively, considering measurement reliability and clinical significance [9- 
11]. The following variables were included as possible confounders: age, sex, body mass index, New York Heart Association class $\geq 3$, Canadian Study of Health and Aging Clinical Frailty Scale (CSHA-CFS) $\geq 5$, Society of Thoracic Surgeons surgical mortality risk score, estimated glomerular filtration rate, post-TAVR new-onset complete left bundle branch block (CLBBB), post-TAVR pacemaker implantation, baseline brain natriuretic peptide (BNP), baseline left ventricular end-diastolic volume, baseline left ventricular ejection fraction (LVEF), baseline aortic valve area (AVA), baseline E/E', baseline peak velocity (Vmax), baseline mean aortic valve pressure gradient (mPG), and baseline late $\mathrm{H} / \mathrm{M}$. Among all variables with $P<0.2$ according to the univariate analysis, clinically important variables with a lower $P$-value were selected as confounders, considering the number of endpoints and multicollinearity.

To further investigate the association between the baseline MPG and mid-term improvement in the late $\mathrm{H} / \mathrm{M}$, the study participants were retrospectively divided into three groups according to the baseline mPG: the low-gradient (LG) group, which consisted of patients with baseline $\mathrm{mPG}<42 \mathrm{mmHg}(n=24)$; the highgradient (HG) group, which comprised patients with $\mathrm{mPG} \geq 42 \mathrm{mmHg}$ and $<58 \mathrm{mmHg}(n=26)$; and the very-high-gradient (VHG) group, which included patients with $\mathrm{mPG} \geq 58 \mathrm{mmHg}(n=25)$. The baseline mPG thresholds were set to ensure an even distribution of the number of patients. Subgroup analyses of changes in the late $\mathrm{H} / \mathrm{M}$ were subsequently performed. To compare the late $\mathrm{H} / \mathrm{M}$ and its changes among these three groups at three time points (at baseline, within 2 weeks after TAVR, and at 6-12 months after TAVR), one-way ANOVA or Kruskal-Wallis test was conducted for normally distributed or non-normally distributed variables, respectively. In case of significance, pairwise post hoc tests with Holm correction were performed.

Statistical analyses were performed using R software packages version 3.1.1 (R Development Core Team, Auckland, New Zealand), with the significance level for statistical hypothesis testing set at 0.05 and with the alternative hypothesis being two-sided.

\section{Results}

\section{Patient Characteristics}

Of 183 consecutive patients who underwent TAVR, 65 were excluded from initial evaluation, whereas 7 were excluded from post-TAVR evaluation due to periprocedural complications. During the follow-up period, 21 patients who required additional medical treatment, 8 patients who were followed up at other hospitals, and 7 patients who died before the final MIBG imaging were excluded from the final MIBG evaluation. The final study population comprised 75 patients (Fig. 1).

The baseline patient characteristics are summarized in Table 1. The median age of the study population was 86 years; 19 patients (25.3\%) were males. A total of 57 patients were classified as having highgradient severe AS (baseline $\mathrm{mPG} \geq 40 \mathrm{mmHg}$ ), whereas 18 patients were deemed to have low-gradient severe AS (baseline $\mathrm{mPG}<40 \mathrm{mmHg}$ ), including 15 patients who were diagnosed with "paradoxical low- 
flow low-gradient severe AS" (LVEF $\geq 50 \%$ ). The median period between TAVR and second MIBG scintigraphy and between TAVR and third MIBG scintigraphy was 6 and 205 days, respectively. 
Table 1

Baseline Characteristics and Procedural Data

\section{Parameters}

\section{Baseline characteristics}

Age, years

Male sex, $n(\%)$

Body mass index, $\mathrm{kg} / \mathrm{m}^{2}$

Society of Thoracic Surgeons score

CSHA-CFS score $\geq 5, n(\%)$

New York Heart Association class III/IV, $n$ (\%)

Current smoker, $n(\%)$

Past medical history, $n(\%)$

Hypertension

59 (78.7)

Dyslipidaemia

Diabetes mellitus

Atrial fibrillation (chronic/paroxysmal)

Previous percutaneous coronary intervention

Previous coronary artery bypass grafting

Previous myocardial infarction

Previous cerebrovascular disease

Pacemaker implantation

Medications, $n(\%)$

ACEls/ARBS

Beta-blockers

Diuretics

11 (14.7)

23 (30.7)

4 (5.3)

8 (10.7)

15 (20.0)

$9(12.0)$

40 (53.3)

$36(48.0)$

39 (52.0)
Total $(n=75)$

86 (84 to 89 )

19 (25.3)

21.6 (19.7 to 24.0 )

5.7 (4.5 to 8.2)

40 (54.1)

24 (32.0)

2 (2.7)

8 (10.7)/9 (12.0)

Categorical and continuous variables are presented as number (percentage) and median (25th-75th percentile), respectively.

ACEls indicate angiotensin-converting enzyme inhibitors; ARBs, angiotensin II receptor blockers; AS, aortic stenosis; CLBBB, complete left bundle branch block; CSHA-CFS, Canadian Study of Health and Aging Clinical Frailty Scale; LVEF, left ventricular ejection fraction; MIBG, ${ }^{123}$-metaiodobenzylguanidine; $\mathrm{mPG}$, mean aortic valve pressure gradient; and TAVR, transcatheter aortic valve replacement. 


\section{Parameters}

AS categories, $n(\%)$

High-gradient severe AS (mPG $\geq 40 \mathrm{mmHg}$ )

Low-gradient severe AS $(\mathrm{mPG}<40 \mathrm{mmHg})$

- Classical low-flow low-gradient severe AS (LVEF < 50\%)

- Paradoxical low-flow low-gradient severe AS (LVEF $\geq 50 \%$ )

Period between TAVR and second MIBG imaging (days)

Period between TAVR and third MIBG imaging (days)
Total $(n=75)$

$57(76.0)$

$18(24.0)$

$3(4.0)$

$15(20.0)$

6 (4 to 8$)$

205 (191 to 222)

\section{Procedural data}

Approach, $n(\%)$

Transfemoral

Trans-subclavian

Anaesthesia, $n(\%)$

General anaesthesia

Conscious sedation with local anaesthesia

Valve type, $n(\%)$

Edwards SAPIEN 3 $36(48.0)$

Medtronic CoreValve Evolut R/Pro $39(52.0)$

Post-TAVR pacemaker implantation, $n(\%)$

Post-TAVR new-onset CLBBB, $n$ (\%)

Categorical and continuous variables are presented as number (percentage) and median (25th-75th percentile), respectively.

ACEls indicate angiotensin-converting enzyme inhibitors; ARBs, angiotensin II receptor blockers; AS, aortic stenosis; CLBBB, complete left bundle branch block; CSHA-CFS, Canadian Study of Health and Aging Clinical Frailty Scale; LVEF, left ventricular ejection fraction; MIBG, ${ }^{123}$-metaiodobenzylguanidine; $\mathrm{mPG}$, mean aortic valve pressure gradient; and TAVR, transcatheter aortic valve replacement.

\section{Changes in Laboratory, Echocardiographic, and MIBG Findings After TAVR}

Serial changes in laboratory, echocardiographic, and MIBG findings are summarized in Table 2. Hemodynamic AS parameters such as Vmax, mPG, and AVA improved immediately after TAVR and were 
well maintained until 6-12 months post-procedurally. The LVEF increased after TAVR and was sustained after 6-12 months. 
Table 2

Laboratory, Echocardiographic, and MIBG Findings at Baseline, $<2$ Weeks After TAVR, and 6-12 Months After TAVR

\begin{tabular}{|c|c|c|c|c|c|c|c|}
\hline Parameters & $\begin{array}{l}\text { A: at } \\
\text { baseline } \\
(n=75)\end{array}$ & $\begin{array}{l}\text { B: at < } 2 \\
\text { weeks } \\
\text { after TAVR } \\
(n=75)\end{array}$ & $\begin{array}{l}\text { C: at } 6-12 \\
\text { months after } \\
\text { TAVR } \\
(n=75)\end{array}$ & $\begin{array}{l}\text { Overall } \\
P \\
\text { value }\end{array}$ & $\begin{array}{l}\text { A vs. } \\
\text { B } \\
P \\
\text { value }\end{array}$ & $\begin{array}{l}\text { B vs. } \\
\text { C } \\
P \\
\text { value }\end{array}$ & $\begin{array}{l}\text { A vs. } \\
\text { C } \\
P \\
\text { value }\end{array}$ \\
\hline \multicolumn{8}{|l|}{ Laboratory data } \\
\hline Haemoglobin, g/dL & $\begin{array}{l}10.7 \\
(10.1 \text { to } \\
12.3)\end{array}$ & $\begin{array}{l}10.2(9.5 \\
\text { to } 11.2)\end{array}$ & $\begin{array}{l}11.3 \text { (10.7 to } \\
12.3)\end{array}$ & $\begin{array}{l}< \\
0.001\end{array}$ & 0.001 & $\begin{array}{l}<.001 \\
0.00\end{array}$ & 0.010 \\
\hline Albumin, $\mathrm{g} / \mathrm{dL}$ & $\begin{array}{l}3.8(3.5 \\
\text { to } 4.1)\end{array}$ & $\begin{array}{l}3.1(2.9 \text { to } \\
3.3)\end{array}$ & $\begin{array}{l}3.9(3.7 \text { to } \\
4.1)\end{array}$ & $<.001$ & $<.001$ & $<.001$ & 0.003 \\
\hline Creatinine, $\mathrm{mg} / \mathrm{dL}$ & $\begin{array}{l}0.83 \\
(0.64 \text { to } \\
1.17)\end{array}$ & $\begin{array}{l}0.77(0.57 \\
\text { to } 0.98)\end{array}$ & $\begin{array}{l}0.91 \text { ( } 0.70 \text { to } \\
1.21)\end{array}$ & $\begin{array}{l}< \\
0.001\end{array}$ & $<001$ & $<0.001$ & 0.003 \\
\hline $\begin{array}{l}\text { eGFR, } \\
\mathrm{mL} / \mathrm{min} / 1.73 \mathrm{~m}^{2}\end{array}$ & $\begin{array}{l}51.5 \\
(38.0 \text { to } \\
66.4)\end{array}$ & $\begin{array}{l}60.4(49.1 \\
\text { to } 75.8)\end{array}$ & $\begin{array}{l}50.3 \text { (35.8 to } \\
61.1)\end{array}$ & $<.001$ & $\begin{array}{l}< \\
0.001\end{array}$ & $<.001$ & 0.020 \\
\hline BNP, $p g / m L$ & $\begin{array}{l}290(133 \\
\text { to } 538)\end{array}$ & $\begin{array}{l}136(74 \text { to } \\
210)\end{array}$ & $\begin{array}{l}89(58 \text { to } \\
209)\end{array}$ & $<.001$ & $<.001$ & 0.030 & $\begin{array}{l}< \\
0.001\end{array}$ \\
\hline \multicolumn{8}{|l|}{$\begin{array}{l}\text { Echocardiographic } \\
\text { data }\end{array}$} \\
\hline Vmax, $\mathrm{m} / \mathrm{s}$ & $\begin{array}{l}4.6(4.1 \\
\text { to } 5.0)\end{array}$ & $\begin{array}{l}2.1(1.8 \text { to } \\
2.4)\end{array}$ & $\begin{array}{l}2.0(1.7 \text { to } \\
2.2)\end{array}$ & $<.001$ & $<.001$ & 0.002 & $\begin{array}{l}< \\
0.001\end{array}$ \\
\hline $\mathrm{mPG}, \mathrm{mmHg}$ & $\begin{array}{l}49 \text { (40 to } \\
61)\end{array}$ & 9 (7 to 12$)$ & 8 (6 to 10$)$ & $<.001$ & $<.001$ & $<001$ & $\begin{array}{l}<.001 \\
0.001\end{array}$ \\
\hline AVA, $\mathrm{cm}^{2}$ & $\begin{array}{l}0.6(0.4 \\
\text { to } 0.7)\end{array}$ & $\begin{array}{l}1.4(1.2 \text { to } \\
1.7)\end{array}$ & $\begin{array}{l}1.6(1.2 \text { to } \\
1.9)\end{array}$ & $\begin{array}{l}< \\
0.001\end{array}$ & $\begin{array}{l}< \\
0.001\end{array}$ & 0.060 & $\begin{array}{l}<.001 \\
0.001\end{array}$ \\
\hline LVESV, mL & $\begin{array}{l}25 \text { (20 to } \\
40)\end{array}$ & $\begin{array}{l}22(18 \text { to } \\
35)\end{array}$ & 22 (18 to 27 ) & 0.002 & 0.002 & 0.069 & $\begin{array}{l}< \\
0.001\end{array}$ \\
\hline LVEDV, mL & $\begin{array}{l}74 \text { (58 to } \\
90)\end{array}$ & $\begin{array}{l}74(58 \text { to } \\
83)\end{array}$ & 70 (54 to 79$)$ & 0.011 & 0.205 & 0.080 & 0.006 \\
\hline
\end{tabular}

Categorical and continuous variables are presented as number (percentage) and median (25th-75th percentile), respectively.

AVA indicates aortic valve area; BNP, brain natriuretic peptide; eGFR, estimated glomerular filtration rate; $E / E^{\prime}$, ratio of early diastolic left transmitral flow velocity to septal mitral annular velocity; $H / M$, heart-mediastinum ratio; LVEDV, left ventricular end-diastolic volume; LVEF, left ventricular ejection fraction; LVESV, left ventricular end-systolic volume; $\mathrm{MPG}$, mean aortic valve pressure gradient; TAVR, transcatheter aortic valve replacement; Vmax, peak velocity; and WR, washout rate. 


\begin{tabular}{|c|c|c|c|c|c|c|c|}
\hline Parameters & $\begin{array}{l}\text { A: at } \\
\text { baseline } \\
(n=75)\end{array}$ & $\begin{array}{l}\text { B: at <2 } \\
\text { weeks } \\
\text { after TAVR } \\
(n=75)\end{array}$ & $\begin{array}{l}\text { C: at } 6-12 \\
\text { months after } \\
\text { TAVR } \\
(n=75)\end{array}$ & $\begin{array}{l}\text { Overall } \\
P \text {. } \\
\text { value }\end{array}$ & $\begin{array}{l}\text { A vs. } \\
\text { B } \\
P \\
\text { value }\end{array}$ & $\begin{array}{l}\text { B vs. } \\
\text { C } \\
P \\
\text { value }\end{array}$ & $\begin{array}{l}\text { A vs. } \\
\text { C } \\
P \\
\text { value }\end{array}$ \\
\hline LVEF, \% & $\begin{array}{l}61 \text { (52 to } \\
69)\end{array}$ & $\begin{array}{l}66 \text { (58 to } \\
71)\end{array}$ & 66 (59 to 69 ) & 0.001 & $<.001$ & 0.969 & 0.005 \\
\hline $\mathrm{E} / \mathrm{E}^{\prime}$ & $\begin{array}{l}27 \text { ( } 20 \text { to } \\
34)\end{array}$ & $\begin{array}{l}26 \text { (21 to } \\
35)\end{array}$ & 24 (17 to 30$)$ & 0.044 & 0.969 & 0.058 & 0.058 \\
\hline \multicolumn{8}{|l|}{ MIBG data } \\
\hline Early H/M & $\begin{array}{l}3.03 \\
(2.66 \text { to } \\
3.25)\end{array}$ & $\begin{array}{l}2.97(2.56 \\
\text { to } 3.16)\end{array}$ & $\begin{array}{l}3.02(2.70 \text { to } \\
3.35)\end{array}$ & 0.233 & - & - & - \\
\hline Late $\mathrm{H} / \mathrm{M}$ & $\begin{array}{l}2.57 \\
(2.12 \text { to } \\
2.85)\end{array}$ & $\begin{array}{l}2.62(2.26 \\
\text { to } 2.95)\end{array}$ & $\begin{array}{l}2.71 \text { ( } 2.39 \text { to } \\
3.04)\end{array}$ & 0.005 & 0.041 & 0.041 & $\begin{array}{l}< \\
0.001\end{array}$ \\
\hline WR, \% & $\begin{array}{l}32.7 \\
(27.3 \text { to } \\
45.5)\end{array}$ & $\begin{array}{l}27.2(22.3 \\
\text { to } 37.1)\end{array}$ & $\begin{array}{l}29.8(22.9 \text { to } \\
35.3)\end{array}$ & $<.001$ & 0.003 & 0.827 & 0.003 \\
\hline \multicolumn{8}{|l|}{$\begin{array}{l}\text { Change from } \\
\text { baseline level }\end{array}$} \\
\hline$\Delta$ Late $\mathrm{H} / \mathrm{M}$ & - & $\begin{array}{l}0.09(-0.11 \\
\text { to } 0.26)\end{array}$ & $\begin{array}{l}0.18(-0.05 \text { to } \\
0.56)\end{array}$ & 0.021 & - & - & - \\
\hline$\Delta \mathrm{WR}, \%$ & - & $\begin{array}{l}-3.3(-7.3 \text { to } \\
1.7)\end{array}$ & $\begin{array}{l}-3.8(-8.3 \text { to } \\
1.6)\end{array}$ & 0.827 & - & - & - \\
\hline $\begin{array}{l}\text { Improvement in } \\
\text { late } \mathrm{H} / \mathrm{M}(>0.1), \%\end{array}$ & - & $34(45.3)$ & $43(57.3)$ & 0.072 & - & - & - \\
\hline $\begin{array}{l}\text { Improvement in WR } \\
(>3 \%), \%\end{array}$ & - & $39(52.0)$ & $40(53.3)$ & 0.835 & - & - & - \\
\hline \multicolumn{8}{|c|}{$\begin{array}{l}\text { Categorical and continuous variables are presented as number (percentage) and median (25th-75th } \\
\text { percentile), respectively. }\end{array}$} \\
\hline \multicolumn{8}{|c|}{$\begin{array}{l}\text { AVA indicates aortic valve area; BNP, brain natriuretic peptide; eGFR, estimated glomerular filtration } \\
\text { rate; E/E', ratio of early diastolic left transmitral flow velocity to septal mitral annular velocity; } \mathrm{H} / \mathrm{M} \text {, } \\
\text { heart-mediastinum ratio; LVEDV, left ventricular end-diastolic volume; LVEF, left ventricular ejection } \\
\text { fraction; LVESV, left ventricular end-systolic volume; MPG, mean aortic valve pressure gradient; TAVR, } \\
\text { transcatheter aortic valve replacement; Vmax, peak velocity; and WR, washout rate. }\end{array}$} \\
\hline
\end{tabular}

Changes in MIBG parameters are presented in a boxplot in Fig. 2. Late $\mathrm{H} / \mathrm{M}$ significantly improved within 2 weeks after TAVR $(P=0.041)$ and further improved over $6-12$ months after TAVR $(P=0.041)$. WR rapidly improved after TAVR $(P=0.003)$ but showed no further improvement over $6-12$ months $(P=$ $0.827)$. Nevertheless, compared to the baseline level, the WR values were still significantly improved at 612 months after TAVR $(P=0.003)$. 
The increase in the late $\mathrm{H} / \mathrm{M}$ at 6-12 months post-TAVR (i.e., $\Delta$ late $\mathrm{H} / \mathrm{M}$ [6-12 months - baseline]) was significantly greater than that just after TAVR (i.e., $\Delta$ late H/M $[<2$ weeks - baseline]) $(P=0.021)$. In contrast, the decrease in the WR at 6-12 months after TAVR (i.e., $\triangle$ WR [6-12 months - baseline]) was not significantly different from that immediately after TAVR (i.e., $\triangle$ WR $[<2$ weeks - baseline]) $(P=0.827)$. No changes in the early $\mathrm{H} / \mathrm{M}$ were observed at the three time points.

\section{Baseline $\mathrm{mPG}$ and Improvement in Late $\mathrm{H} / \mathrm{M}$ at 6-12 Months After TAVR}

Overall, 43 out of 75 patients showed improvement in the late $\mathrm{H} / \mathrm{M}(>0.1)$ at $6-12$ months after TAVR. Multivariate analysis revealed that baseline $\mathrm{MPG}$ was associated with such mid-term improvement in the late $\mathrm{H} / \mathrm{M}$ (adjusted odds ratio: $0.035 ; 95 \%$ confidence interval: $0.004-0.070 ; P=0.037$ ) (Table 3). 
Table 3

Predictors of Improvement in Late H/M (>0.1) at 6-12 Months After TAVR Compared to the Baseline Level

\begin{tabular}{|c|c|c|c|c|}
\hline \multirow[t]{2}{*}{ Parameters } & \multicolumn{2}{|l|}{ Univariate } & \multicolumn{2}{|l|}{ Multivariate } \\
\hline & OR $(95 \% \mathrm{Cl})$ & $\begin{array}{l}\mathrm{P}- \\
\text { value }\end{array}$ & $\begin{array}{l}\text { Adjusted OR (95\% } \\
\text { Cl) }\end{array}$ & $\begin{array}{l}\mathrm{P} \text { - } \\
\text { value }\end{array}$ \\
\hline Age (per 1 year) & $\begin{array}{l}0.082(-0.040 \text { to } \\
0.215)\end{array}$ & 0.200 & & \\
\hline Sex & $\begin{array}{l}-0.256(-1.309 \text { to } \\
0.804)\end{array}$ & 0.632 & & \\
\hline Body mass index & $\begin{array}{l}-0.067(-0.208 \text { to } \\
0.067)\end{array}$ & 0.329 & & \\
\hline $\begin{array}{l}\text { New York Heart Association } \\
\text { class } \geq \text { III }\end{array}$ & $\begin{array}{l}0.848(-0.161 \text { to } \\
1.936)\end{array}$ & 0.109 & & \\
\hline CSHA-CFS score $\geq 5$ & $\begin{array}{l}0.967(0.033 \text { to } \\
1.936)\end{array}$ & 0.045 & $\begin{array}{l}0.810(-0.191 \text { to } \\
1.839)\end{array}$ & 0.115 \\
\hline $\begin{array}{l}\text { Society of Thoracic Surgeons } \\
\text { score }\end{array}$ & $\begin{array}{l}0.062(-0.113 \text { to } \\
0.246)\end{array}$ & 0.492 & & \\
\hline eGFR & $\begin{array}{l}-0.007(-0.030 \text { to } \\
0.015)\end{array}$ & 0.526 & & \\
\hline Post-TAVR new-onset CLBBB & $\begin{array}{l}0.049(-1.197 \text { to } \\
1.357)\end{array}$ & 0.939 & & \\
\hline $\begin{array}{l}\text { Post-TAVR pacemaker } \\
\text { implantation }\end{array}$ & $\begin{array}{l}-1.792(-4.793 \text { to } \\
0.181)\end{array}$ & 0.117 & & \\
\hline Baseline BNP & $\begin{array}{l}0.001(0.0002 \text { to } \\
0.003)\end{array}$ & 0.082 & $\begin{array}{l}0.001 \text { ( } 0.0001 \text { to } \\
0.003)\end{array}$ & 0.067 \\
\hline$\triangle \mathrm{BNP}$ (6-12 months - baseline) & $\begin{array}{l}-0.002(-0.004 \text { to } \\
-0.0002)\end{array}$ & 0.089 & & \\
\hline Baseline LVEDV & $\begin{array}{l}0.016(-0.003 \text { to } \\
0.036)\end{array}$ & 0.113 & & \\
\hline Baseline LVEF & $\begin{array}{l}-0.012(-0.053 \text { to } \\
0.028)\end{array}$ & 0.569 & & \\
\hline
\end{tabular}

In the multivariate model, the adjusted $\mathrm{OR}$ for the improvement in late $\mathrm{H} / \mathrm{M}>0.1$ at $6-12$ months after TAVR, as compared to that for the improvement in late $\mathrm{H} / \mathrm{M} \leq 0.1$, was calculated by adjusting the following variables: CSHA-CFS score $\geq 5$, baseline BNP level, and baseline MPG.

AVA indicates aortic valve area; $\mathrm{BNP}$, brain natriuretic peptide; $\mathrm{Cl}$, confidence interval; $\mathrm{CLBBB}$, complete left bundle branch block; CSHA-CFS, Canadian Study of Health and Aging Clinical Frailty Scale; eGFR, estimated glomerular filtration rate; $E / E^{\prime}$, ratio of early diastolic left transmitral flow velocity to septal mitral annular velocity; $\mathrm{H} / \mathrm{M}$, heart-mediastinum ratio; LVEDV, left ventricular end-diastolic volume; LVEF, left ventricular ejection fraction; OR, odds ratio; $\mathrm{mPG}$, mean aortic valve pressure gradient; TAVR, transcatheter aortic valve replacement; and Vmax, peak velocity. 


\begin{tabular}{|c|c|c|c|c|}
\hline \multirow[t]{2}{*}{ Parameters } & \multicolumn{2}{|l|}{ Univariate } & \multicolumn{2}{|l|}{ Multivariate } \\
\hline & OR $(95 \% \mathrm{Cl})$ & $\begin{array}{l}\mathrm{P}- \\
\text { value }\end{array}$ & $\begin{array}{l}\text { Adjusted OR (95\% } \\
\text { Cl) }\end{array}$ & $\begin{array}{l}\mathrm{P} \text { - } \\
\text { value }\end{array}$ \\
\hline$\Delta$ LVEF (6-12 months - baseline) & $\begin{array}{l}0.010(-0.026 \text { to } \\
0.047)\end{array}$ & 0.582 & & \\
\hline Baseline AVA & $\begin{array}{l}0.272(-2.845 \text { to } \\
3.427)\end{array}$ & 0.864 & & \\
\hline Baseline E/E' & $\begin{array}{l}0.025(-0.019 \text { to } \\
0.074)\end{array}$ & 0.276 & & \\
\hline Baseline Vmax & $\begin{array}{l}0.493(-0.170 \text { to } \\
1.217)\end{array}$ & 0.157 & & \\
\hline Baseline mPG & $\begin{array}{l}0.031 \text { ( }(0.002 \text { to } \\
0.062)\end{array}$ & 0.043 & $\begin{array}{l}0.035 \text { (0.004 to } \\
0.070)\end{array}$ & 0.037 \\
\hline$\triangle \mathrm{mPG}$ (6-12 months - baseline) & $\begin{array}{l}-0.030(-0.061 \text { to } \\
-0.001)\end{array}$ & 0.049 & & \\
\hline Baseline late $\mathrm{H} / \mathrm{M}$ & $\begin{array}{l}-0.228(-1.060 \text { to } \\
0.574)\end{array}$ & 0.575 & & \\
\hline \multicolumn{5}{|c|}{$\begin{array}{l}\text { In the multivariate model, the adjusted } \mathrm{OR} \text { for the improvement in late } \mathrm{H} / \mathrm{M}>0.1 \text { at } 6-12 \text { months after } \\
\text { TAVR, as compared to that for the improvement in late } \mathrm{H} / \mathrm{M} \leq 0.1 \text {, was calculated by adjusting the } \\
\text { following variables: CSHA-CFS score } \geq 5 \text {, baseline BNP level, and baseline mPG. }\end{array}$} \\
\hline \multicolumn{5}{|c|}{$\begin{array}{l}\text { AVA indicates aortic valve area; BNP, brain natriuretic peptide; Cl, confidence interval; CLBBB, complete } \\
\text { left bundle branch block; CSHA-CFS, Canadian Study of Health and Aging Clinical Frailty Scale; eGFR, } \\
\text { estimated glomerular filtration rate; E/E', ratio of early diastolic left transmitral flow velocity to septal } \\
\text { mitral annular velocity; H/M, heart-mediastinum ratio; LVEDV, left ventricular end-diastolic volume; } \\
\text { LVEF, left ventricular ejection fraction; OR, odds ratio; mPG, mean aortic valve pressure gradient; TAVR, } \\
\text { transcatheter aortic valve replacement; and Vmax, peak velocity. }\end{array}$} \\
\hline
\end{tabular}

The late $\mathrm{H} / \mathrm{M}$ and associated clinical parameters in the three groups who were categorized according to the baseline $\mathrm{mPG}$ (LG group, < $42 \mathrm{mmHg}$; HG group, $\geq 42 \mathrm{mmHg}$ and < $58 \mathrm{mmHg}$; and VHG group, $\geq 58$ $\mathrm{mmHg}$ ) are presented in Table 4. No significant difference in baseline LVEF was observed among the three groups. In the LG group, 21 (87.5\%) out of 24 patients had baseline LVEF $\geq 50 \%$. The increase in late $\mathrm{H} / \mathrm{M}$ within 2 weeks after TAVR (i.e., $\Delta$ late H/M [<2 weeks - baseline]) was comparable among the three groups, whereas the increase in late $\mathrm{H} / \mathrm{M}$ at 6-12 months after TAVR (i.e., $\Delta$ late $\mathrm{H} / \mathrm{M}$ [6-12 months - baseline]) tended to be greater in the HG group than in the LG group and in the VHG group than in the HG group. The VHG group had significantly higher $\Delta$ late H/M (6-12 months - baseline) than the LG group ( $P$ $=0.029)$ (Fig. 3). 
Table 4

Late $\mathrm{H} / \mathrm{M}$ and Associated Clinical Parameters in the Three Groups Categorized According to Baseline mPG: Low mPG, High $\mathrm{mPG}$, and Very High $\mathrm{mPG}$

\begin{tabular}{|c|c|c|c|c|c|c|c|}
\hline Parameters & $\begin{array}{l}\text { A: low } \\
\text { mPG } \\
(n=24)\end{array}$ & $\begin{array}{l}\text { B: high } \\
\text { mPG } \\
(n=26)\end{array}$ & $\begin{array}{l}\text { C: very } \\
\text { high mPG } \\
(n=25)\end{array}$ & $\begin{array}{l}\text { Overall } \\
P \text {. } \\
\text { value }\end{array}$ & $\begin{array}{l}\text { Avs. } \\
\text { B } \\
P \\
\text { value }\end{array}$ & $\begin{array}{l}\text { B vs. } \\
\text { C } \\
P \\
\text { value }\end{array}$ & $\begin{array}{l}\text { A vs. } \\
\text { C } \\
P \\
\text { value }\end{array}$ \\
\hline \multicolumn{8}{|l|}{ Laboratory data } \\
\hline Baseline BNP, pg/mL & $\begin{array}{l}194(45 \\
\text { to } 437)\end{array}$ & $\begin{array}{l}269(125 \\
\text { to } 555)\end{array}$ & $\begin{array}{l}365(211 \\
\text { to } 574)\end{array}$ & 0.115 & - & - & - \\
\hline \multicolumn{8}{|l|}{$\begin{array}{l}\text { Echocardiographic } \\
\text { data }\end{array}$} \\
\hline Baseline Vmax, m/s & $\begin{array}{l}4.1 \text { (3.8 to } \\
4.1)\end{array}$ & $\begin{array}{l}4.6(4.3 \text { to } \\
4.7)\end{array}$ & $\begin{array}{l}5.2(5.0 \text { to } \\
5.7)\end{array}$ & $<.001$ & $<.001$ & $<001$ & $\begin{array}{l}< \\
0.001\end{array}$ \\
\hline Baseline mPG, mmHg & $\begin{array}{l}38 \text { (33 to } \\
39)\end{array}$ & $\begin{array}{l}49 \text { (44 to } \\
53)\end{array}$ & $\begin{array}{l}69(61 \text { to } \\
80)\end{array}$ & $<.001$ & $<.001$ & $<001$ & $\begin{array}{l}< \\
0.001\end{array}$ \\
\hline Baseline AVA, $\mathrm{cm}^{2}$ & $\begin{array}{l}0.6(0.5 \text { to } \\
0.7)\end{array}$ & $\begin{array}{l}0.6(0.5 \text { to } \\
0.7)\end{array}$ & $\begin{array}{l}0.5(0.4 \text { to } \\
0.5)\end{array}$ & 0.003 & 0.828 & 0.007 & 0.006 \\
\hline Baseline LVEF, \% & $\begin{array}{l}61(53 \text { to } \\
69)\end{array}$ & $\begin{array}{l}64(57 \text { to } \\
69)\end{array}$ & $\begin{array}{l}58(50 \text { to } \\
64)\end{array}$ & 0.204 & - & - & - \\
\hline $\begin{array}{l}\text { Baseline LVEF > 50\%, } n \\
(\%)\end{array}$ & $21(87.5)$ & $23(88.5)$ & $19(76.0)$ & 0.492 & - & - & - \\
\hline \multicolumn{8}{|l|}{ MIBG data } \\
\hline Late $\mathrm{H} / \mathrm{M}$ at baseline & $\begin{array}{l}2.69(2.19 \\
\text { to } 3.00)\end{array}$ & $\begin{array}{l}2.54(2.02 \\
\text { to } 2.90)\end{array}$ & $\begin{array}{l}2.53(2.12 \\
\text { to } 2.69)\end{array}$ & 0.441 & - & - & - \\
\hline $\begin{array}{l}\text { Late } \mathrm{H} / \mathrm{M} \text { at }<2 \text { weeks } \\
\text { after TAVR }\end{array}$ & $\begin{array}{l}2.67(2.36 \\
\text { to } 3.01)\end{array}$ & $\begin{array}{l}2.77(1.93 \\
\text { to } 2.97)\end{array}$ & $\begin{array}{l}2.57(2.19 \\
\text { to } 2.65)\end{array}$ & 0.404 & - & - & - \\
\hline $\begin{array}{l}\text { Late } \mathrm{H} / \mathrm{M} \text { at } 6-12 \\
\text { months after TAVR }\end{array}$ & $\begin{array}{l}2.61(2.34 \\
\text { to } 3.00)\end{array}$ & $\begin{array}{l}2.70(2.06 \\
\text { to } 3.03)\end{array}$ & $\begin{array}{l}2.82(2.56 \\
\text { to } 3.04)\end{array}$ & 0.356 & - & - & - \\
\hline $\begin{array}{l}\Delta \text { Late } \mathrm{H} / \mathrm{M}(<2 \text { weeks - } \\
\text { baseline })\end{array}$ & $\begin{array}{l}0.09 \\
(-0.08 \text { to } \\
0.25)\end{array}$ & $\begin{array}{l}0.11 \\
(-0.05 \text { to } \\
0.26)\end{array}$ & $\begin{array}{l}-0.01 \\
(-0.20 \text { to } \\
0.22)\end{array}$ & 0.424 & - & - & - \\
\hline $\begin{array}{l}\Delta \text { Late } \mathrm{H} / \mathrm{M}(6-12 \\
\text { months - baseline })\end{array}$ & $\begin{array}{l}0.01 \\
(-0.25 \text { to } \\
0.34)\end{array}$ & $\begin{array}{l}0.17 \\
(-0.04 \text { to } \\
0.52)\end{array}$ & $\begin{array}{l}0.24(0.07 \\
\text { to } 0.68)\end{array}$ & 0.031 & 0.151 & 0.376 & 0.029 \\
\hline
\end{tabular}

Continuous variables are presented as number (percentage) and median (25th-75th percentile), respectively.

$\mathrm{H} / \mathrm{M}$ indicates heart-mediastinum ratio; MIBG, ${ }^{123}$-meta-iodobenzylguanidine; $\mathrm{MPG}$, mean aortic valve pressure gradient; and TAVR, transcatheter aortic valve replacement 


\begin{tabular}{|c|c|c|c|c|c|c|c|}
\hline Parameters & $\begin{array}{l}\text { A: low } \\
\text { mPG } \\
(n=24)\end{array}$ & $\begin{array}{l}\text { B: high } \\
\text { mPG } \\
(n=26)\end{array}$ & $\begin{array}{l}\text { C: very } \\
\text { high mPG } \\
(n=25)\end{array}$ & $\begin{array}{l}\text { Overall } \\
P \\
\text { value }\end{array}$ & $\begin{array}{l}\text { A vs. } \\
\text { B } \\
P \\
\text { value }\end{array}$ & $\begin{array}{l}\text { B vs. } \\
\text { C } \\
P \\
\text { value }\end{array}$ & $\begin{array}{l}\text { A vs. } \\
\mathrm{C} \\
P \\
\text { value }\end{array}$ \\
\hline $\begin{array}{l}\text { TAVR and second } \\
\text { MIBG (days) }\end{array}$ & 6 (5 to 7 ) & 6 (3 to 7$)$ & 7 (4 to 8) & 0.154 & - & - & - \\
\hline $\begin{array}{l}\text { TAVR and third MIBG } \\
\text { (days) }\end{array}$ & $\begin{array}{l}213(191 \\
\text { to } 231)\end{array}$ & $\begin{array}{l}203(197 \\
\text { to } 218)\end{array}$ & $\begin{array}{l}205(190 \\
\text { to } 217)\end{array}$ & 0.781 & - & - & - \\
\hline \multicolumn{8}{|c|}{$\begin{array}{l}\text { Continuous variables are presented as number (percentage) and median ( } 25 \text { th-75th percentile), } \\
\text { respectively. }\end{array}$} \\
\hline
\end{tabular}

\section{Discussion}

The present study yielded the following two main findings: (1) CSNF, as denoted by late H/M, demonstrated a sustained improvement from within 2 weeks after TAVR until 6-12 months later, and (2) higher baseline $\mathrm{mPG}$ was an independent predictor of mid-term improvement in late H/M after TAVR. As no studies have evaluated the long-term (> 6 months) effects of TAVR on CSNF, our study could provide physicians with new insights into this field.

\section{Mid-Term Effects of TAVR on CSNF}

In our results, late $\mathrm{H} / \mathrm{M}$ exhibited a sustained improvement from within 2 weeks after TAVR until 6-12 months later, whereas WR displayed a rapid improvement just after TAVR but remained unchanged over the succeeding 6-12 months. Early H/M represents the amount of cardiac sympathetic nervous terminal endings and uptake-1 function, whereas WR represents sympathetic nervous tone [12]; in comparison, late $\mathrm{H} / \mathrm{M}$ represents both factors. In general, the recovery of the amount of sympathetic nervous terminal endings and uptake- 1 function is expected to require more than a few months, whereas sympathetic nervous tone can rapidly respond to hemodynamic changes and is susceptible to other clinical factors. The present results indicate a gradual trend towards improvement, as demonstrated by the late $\mathrm{H} / \mathrm{M}$, and a rapid but non-continuous improvement in the WR, which were thought to be consistent with the theories that have been stated [12].

\section{Predictors of Improvement in Late H/M at 6-12 Months After TAVR}

A higher baseline $\mathrm{MPG}$ was an independent predictor of mid-term improvement in the late $\mathrm{H} / \mathrm{M}$ after TAVR; in particular, patients with a very high baseline $\mathrm{mPG}(\geq 58 \mathrm{mmHg})$ experienced significantly greater increase in the late $\mathrm{H} / \mathrm{M}$ than those with a low baseline $\mathrm{mPG}(<42 \mathrm{mmHg})$. 
The MPG assessed by Doppler echocardiography is one of the most powerful markers of AS severity in patients with normal cardiac output and preserved LVEF. High MPG is an independent predictor of mortality in patients with severe AS [13]. Moreover, patients with "very severe AS," defined as MPG $\geq 60$ $\mathrm{mmHg}$ and/or $V \max \geq 5 \mathrm{~m} / \mathrm{s}$, are considered at high risk, and current Japanese guidelines recommend early surgery, even when these patients are asymptomatic [14]. In our present study, patients with a very high $\mathrm{mPG}$ ( $\geq 58 \mathrm{mmHg}$ ) exhibited a higher degree of improvement in CSNF after TAVR, suggesting that patients with a very high baseline MPG would gain the greatest prognostic benefit from TAVR, considering the association of TAVR-related improvement in the late $\mathrm{H} / \mathrm{M}$ with post-TAVR prognosis [15].

The effects of TAVR on patients with a low MPG should be carefully discussed. In this study, the increase in the late $\mathrm{H} / \mathrm{M}$ at 6-12 months after TAVR (i.e., $\Delta$ late H/M [6-12 months - baseline]) was minimal among patients with a low baseline $\mathrm{mPG}(<42 \mathrm{mmHg})$. According to current guidelines [14,16], AS with low mPG, so-called "low-flow low-gradient" (LF-LG) severe AS can be divided into two groups-namely, classical LF-LG AS with LVEF $<50 \%$ and paradoxical LF-LG AS with LVEF $\geq 50 \%$. The prognosis of patients with paradoxical LF-LG AS remains controversial, with some studies reporting poor prognosis $[17,18]$ and others indicating good prognosis $[19,20]$. In the present study, most patients with a low mPG ( $<42 \mathrm{mmHg}$ ) were classified as having paradoxical LF-LG AS. Therefore, the present results may represent poor CSNF response after TAVR among patients with paradoxical LF-LG AS, suggesting that patients with paradoxical LF-LG AS may gain benefit from TAVR with respect to symptomatic relief but not in terms of prognostic improvement.

\section{Clinical Implications}

The clinical outcomes of TAVR have improved due to device development and technical advancement, and its application has expanded to include younger patients as well as those with lower surgical risk [2]. Consequently, simpler, easier, and more accurate prognostic indicators are desired. Furthermore, there is an increased need for the appropriate selection of patients who would most benefit from TAVR. In our previous study, we demonstrated that CSNF assessment with MIBG imaging could provide essential information about patients' prognosis after TAVR and that patients exhibiting early improvement in late $\mathrm{H} / \mathrm{M}$ after TAVR had a significantly lower incidence of cardiac events than those without such improvement [15]. The present results indicate that late $\mathrm{H} / \mathrm{M}$ displayed a clearer trend towards improvement at 6-12 months than immediately after TAVR. Because it is assumed that MIBG parameters immediately after TAVR will inevitably be influenced by perioperative procedural factors such as infusion, a comparison of baseline and 6-12-month data may provide a better prognostic indicator for patients who have undergone TAVR. Furthermore, our results suggest that more careful consideration may be given to the indications for TAVR among patients with a low baseline $\mathrm{MPG}$, particularly those with paradoxical LF-LG AS. Serial CSNF assessment using MIBG imaging may be useful in validating TAVR treatment for this AS category.

\section{Study Limitations}


The present study has certain limitations. First, the relatively small sample size of this study may be insufficient to fully reveal the long-term effects on CSNF by TAVR procedure. Second, the prognostic benefit from the improvement in CSNF at 6-12 months after TAVR remains unclear because the patients' prognostic information was not included in the present study. However, because the association of early TAVR-related improvement in the late $\mathrm{H} / \mathrm{M}$ with better prognosis has previously been confirmed [15], the increase in the late H/M revealed in this study should also represent good clinical outcomes after TAVR. Third, there may be some selection bias in sampling, as baseline MIBG imaging could not be performed for critically ill patients with unstable general conditions. Fourth, as this study included Japanese patients only, our results cannot be generalized to other ethnicities.

\section{Conclusion}

CSNF, as denoted by late $\mathrm{H} / \mathrm{M}$, demonstrated a sustained improvement from within 2 weeks after TAVR until 6-12 months later. Such improvement was related to baseline hemodynamic AS severity. Patients with very high baseline $\mathrm{MPG}$ would gain the greatest prognostic benefit from TAVR.

\section{Declarations}

\section{Disclosure of Potential Conflicts of Interest}

Funding: This study was not supported by any funding.

Conflict of Interest: The authors declare that they have no conflict of interest.

\section{Research involving Human Participants and/or Animals}

Ethical approval: All procedures were performed in accordance with the ethical standards of the institutional research committee of Kyoto Prefectural University of Medicine (no. ERB-C-1081-3) and with the 1964 Declaration of Helsinki and its later amendments or comparable ethical standards.

\section{Informed Consent}

Informed consent was obtained from all individual participants included in the study.

\section{Data availability}

The data will be shared on reasonable request to the corresponding author.

\section{Author contributions}

Yoshito Kadoya collected the data, performed statistical analysis, and wrote the manuscript. Kan Zen, Nagara Tamaki, Michiyo Yamano, Tetsuhiro Yamano, Takeshi Nakamura, and Satoaki Matoba revised the manuscript and figures. Shunsuke Nakamura, Tomoki Fujimoto, Masaki Yashige, Kazuaki Takamatsu, 
and Nobuyasu Ito collected the data and revised the manuscript. Hidetake Kawajiri, Satoshi Numata, and Hitoshi Yaku revised the manuscript. All authors read and approved the final manuscript.

Acknowledgments: None

\section{References}

1. Otto $\mathrm{CM}$, Prendergast B. Aortic-valve stenosis--from patients at risk to severe valve obstruction. N Engl J Med. 2014;371:744-56. https://doi.org/10.1056/nejmra1313875

2. Otto CM, Nishimura RA, Bonow RO, Carabello BA, Erwin JP 3rd, Gentile F, Jneid H, Krieger EV, Mack M, McLeod C, et al. 2020 ACC/AHA guideline for the management of patients with valvular heart disease: executive summary: a report of the American College of Cardiology/American Heart Association Joint Committee on Clinical Practice Guidelines. Circulation. 2021;143:e35-71. https://doi.org/10.1161/cir.0000000000000932

3. Sobajima M, Ueno H, Onoda H, Kuwahara H, Tanaka S, Ushijima R, Fukuda N, Yokoyama S, Nagura $S$, Doi T, et al. Transcatheter aortic valve implantation improves cardiac sympathetic nerve activity on ${ }^{123}$-metaiodobenzylguanidine myocardial scintigraphy in severe aortic valve stenosis. Circ J. 2018;82:579-85. https://doi.org/10.1253/circj.cj-17-0817

4. Kadoya Y, Zen K, Tamaki N, Ito N, Kuwabara K, Yamano M, Yamano T, Nakamura T, Matsushima S, Oka K, et al. Early effects of transcatheter aortic valve replacement on cardiac sympathetic nervous function assessed by ${ }^{123}$ I-metaiodobenzylguanidine scintigraphy in patients with severe aortic valve stenosis. Eur J Nucl Med Mol Imaging. 2020;47:1657-67. https://doi.org/10.1007/s00259-01904523-0

5. Cohen-Solal A, Rouzet F, Berdeaux A, Le Guludec D, Abergel E, Syrota A, Merlet P. Effects of carvedilol on myocardial sympathetic innervation in patients with chronic heart failure. J Nucl Med. 2005;46:1796-803.

6. Takeishi Y, Atsumi H, Fujiwara S, Takahashi K, Tomoike H. ACE inhibition reduces cardiac iodine-123MIBG release in heart failure. J Nucl Med. 1997;38:1085-9.

7. Jacobson AF, Travin MI. Impact of medications on mIBG uptake, with specific attention to the heart: comprehensive review of the literature. J Nucl Cardiol. 2015;22:980-93. https://doi.org/10.1007/s12350-015-0170-z

8. Okuda K, Nakajima K, Hosoya T, Ishikawa T, Konishi T, Matsubara K, Matsuo S, Kinuya S. Semiautomated algorithm for calculating heart-to-mediastinum ratio in cardiac iodine-123 MIBG imaging. J Nucl Cardiol. 2011;18:82-89. https://doi.org/10.1007/s12350-010-9313-4

9. Nakata T, Nakajima K, Yamashina S, Yamada T, Momose M, Kasama S, Matsui T, Matsuo S, Travin MI, Jacobson AF. A pooled analysis of multicenter cohort studies of (123)I-mIBG imaging of sympathetic innervation for assessment of long-term prognosis in heart failure. JACC Cardiovasc Imaging. 2013;6:772-84. https://doi.org/10.1016/j.jcmg.2013.02.007 
10. Veltman CE, Boogers MJ, Meinardi JE, Al Younis I, Dibbets-Schneider P, Van der Wall EE, Bax JJ, Scholte AJ. Reproducibility of planar (123)I-meta-iodobenzylguanidine (MIBG) myocardial scintigraphy in patients with heart failure. Eur J Nucl Med Mol Imaging. 2012;39:1599-608. https://doi.org/10.1007/s00259-012-2180-2

11. Kasama S, Toyama T, Sumino H, Nakazawa M, Matsumoto N, Sato Y, Kumakura H, Takayama Y, Ichikawa S, Suzuki T, et al. Prognostic value of serial cardiac ${ }^{123}$ I-MIBG imaging in patients with stabilized chronic heart failure and reduced left ventricular ejection fraction. J Nucl Med. 2008;49:907-14. https://doi.org/10.2967/jnumed.107.047548

12. Carrió I, Cowie MR, Yamazaki J, Udelson J, Camici PG. Cardiac sympathetic imaging with mIBG in heart failure. JACC Cardiovasc Imaging. 2010;3:92-100. https://doi.org/10.1016/j.jcmg.2009.07.014

13. Bohbot Y, Kowalski C, Rusinaru D, Ringle A, Marechaux S, Tribouilloy C. Impact of mean transaortic pressure gradient on long-term outcome in patients with severe aortic stenosis and preserved left ventricular ejection fraction. J Am Heart Assoc. 2017;6:e005850. https://doi.org/10.1161/jaha.117.005850

14. Izumi C, Eishi K, Ashihara K, Arita T, Otsuji Y, Kunihara T, Komiya T, Shibata T, Seo Y, Daimon M, et al. JCS/JSCS/JATS/JSVS 2020 guidelines on the management of valvular heart disease. Circ J. 2020;84:2037-119. https://doi.org/10.1253/circj.cj-20-0135

15. Kadoya Y, Zen K, Tamaki N, Yashige M, Takamatsu K, Ito N, Kuwabara K, Yamano M, Yamano T, Nakamura T, et al. Prognostic value of cardiac ${ }^{123}$-metaiodobenzylguanidine imaging for predicting cardiac events after transcatheter aortic valve replacement. ESC Heart Fail. 2021;8:1106-16. https://doi.org/10.1002/ehf2.13123

16. Baumgartner H, Falk V, Bax JJ, De Bonis M, Hamm C, Holm PJ, lung B, Lancellotti P, Lansac E, Rodriguez Muñoz D, et al. 2017 ESC/EACTS guidelines for the management of valvular heart disease. Eur Heart J. 2017;38:2739-91. https://doi.org/10.1093/eurheartj/ehx391

17. Hachicha Z, Dumesnil JG, Bogaty P, Pibarot P. Paradoxical low-flow, low-gradient severe aortic stenosis despite preserved ejection fraction is associated with higher afterload and reduced survival. Circulation. 2007;115:2856-64. https://doi.org/10.1161/circulationaha.106.668681

18. Jander N, Minners J, Holme I, Gerdts E, Boman K, Brudi P, Chambers JB, Egstrup K, Kesäniemi YA, Malbecq W, et al. Outcome of patients with low-gradient "severe" aortic stenosis and preserved ejection fraction. Circulation. 2011;123:887-95. https://doi.org/10.1161/circulationaha.110.983510

19. Tribouilloy C, Rusinaru D, Maréchaux S, Castel AL, Debry N, Maizel J, Mentaverri R, Kamel S, Slama M, Lévy F. Low-gradient, low-flow severe aortic stenosis with preserved left ventricular ejection fraction: characteristics, outcome, and implications for surgery. J Am Coll Cardiol. 2015;65:55-66. https://doi.org/10.1016/j.jacc.2014.09.080

20. Yamashita E, Takeuchi M, Seo Y, Izumo M, Ishizu T, Sato K, Suzuki K, Akashi YJ, Aonuma K, Otsuji Y, et al. Prognostic value of paradoxical low-gradient severe aortic stenosis in Japan: Japanese Multicenter Aortic Stenosis Study, Retrospective (JUST-R) Registry. J Cardiol. 2015;65:360-8. https://doi.org/10.1016/j.jjcc.2014.12.019 


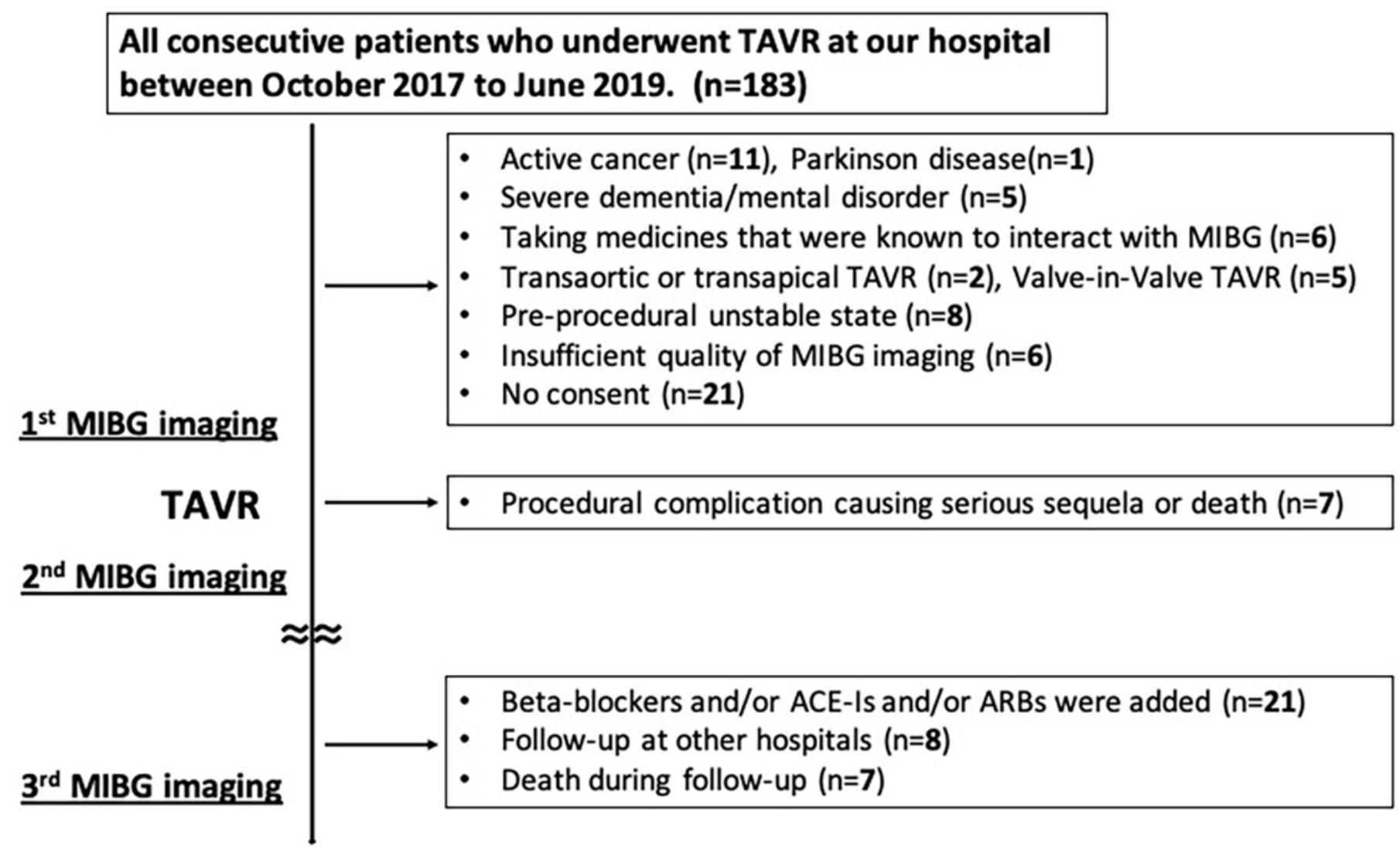

Final study population $(n=75)$

Figure 1

Flow chart illustrating patient selection ACEls indicate angiotensin-converting enzyme inhibitors; ARBs, angiotensin II receptor blockers; MIBG, 123I-meta-iodobenzylguanidine; and TAVR, transcatheter aortic valve replacement.
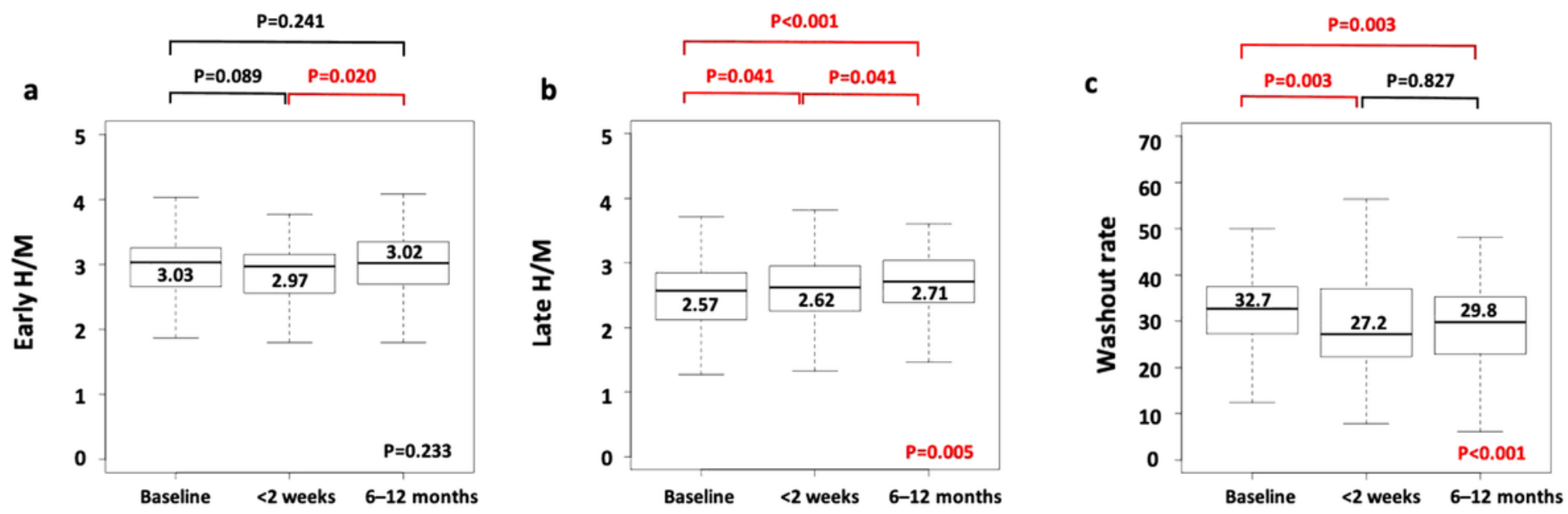
Figure 2

Changes in 123I-meta-iodobenzylguanidine parameters at baseline, $<2$ weeks after transcatheter aortic valve replacement (TAVR), and 6-12 months after TAVR (A) Early $H / M,(B)$ late $H / M$, and (C) WR. $H / M$ indicates heart-mediastinum ratio; and WR, washout ratio.

\section{$P=0.029$}

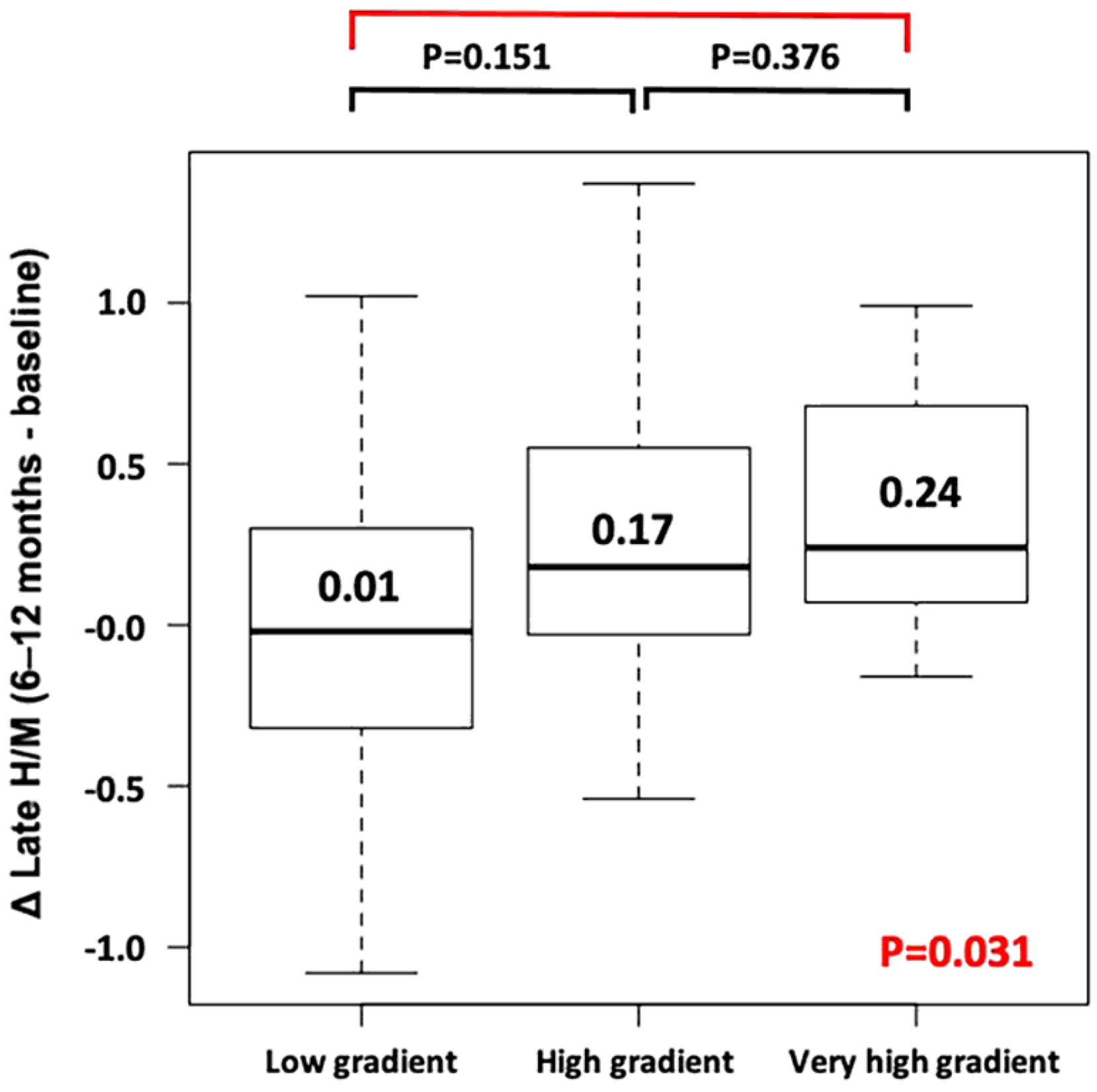

Figure 3

Increase in the late $\mathrm{H} / \mathrm{M}$ at 6-12 months after transcatheter aortic valve replacement (i.e., $\Delta$ late $\mathrm{H} / \mathrm{M}$ [612 months - baseline]) in three groups classified according to the baseline MPG H/M indicates heart- 
mediastinum ratio; $\mathrm{MPG}$, mean aortic valve pressure gradient; and TAVR, transcatheter aortic valve replacement. 